\title{
HEREDITARY MULTIPLE EXOSTOSIS: THE ROLE OF THE MASTOLOGIST IN AN INTERDISCIPLINARY APPROACH
}

Glaucia Mesquita Cordeiro¹, Katrine Evelen Carole Silva Sousa Cançado¹, Nilo Antunes Souza Filho, Jessica Mendes Costa Freitas Santos ${ }^{1}$, José Pereira Guara ${ }^{1}$

${ }^{1}$ Hospital do Câncer Aldenora Bello - São Luis (MA), Brazil.

Introduction: Hereditary Multiple Exostosis (HME) is a disorder of the bone metaphysis, transmitted in an autosomal dominant manner, which is genetically heterogeneous and has incomplete penetration in females, characterized by the presence of multiple exostoses (osteochondromas). It can lead to anatomical and functional impairments for the patient that will remain throughout his life, requiring periodic screening of the lesions to identify and treat them early. Although it can be asymptomatic, a wide spectrum of clinical manifestations are found in pediatric patients with this disorder; the therapeutic approach is substantially surgical, while the clinic is still on an experimental level. The questionable pathophysiological aspects of HME remain a subject of study and controversy in the conduct of therapy and treatment. The diagnosis requires regular screening of the lesions through imaging tests, as well as clinical findings such as: pain, which is the main surgical indication; limitation of movement (for example, forearm rotation may be limited by exostoses between the radius and ulna or maximum knee flexion may be limited by exostosis in the popliteal region) and growth disorders. In this work, we will present a case of osteochondroma in rib with invasion of the left breast in a patient with HME, while emphasizing the role of mastology to prevent compromises and preserve breast aesthetics. Case report: A 14-year-old patient, seen at a tertiary hospital in São Luís, with complaints of pain and hardened tumor lesion in the left breast, diagnosed with osteochondroma. The treatment required the performance of a team composed of a thoracic surgeon and a mastologist to obtain results without functional and aesthetic compromise for the patient. The patient had as main complaint the limitation of movement, dyspnea and pain in the region of the lesion. She underwent a surgical procedure that consisted of an incision through the infra-mammary fold, detachment of the gland by the subfascial plane, opening of the pectoralis major muscle, approach of the rib and resection of the lesion without opening the pleura by the thoracic surgeon. The reconstruction of the breast defect was made with local flaps and closed by planes maintaining an adequate cosmetic and functional breast result. In clinical evaluation after the procedure, she had no complaints and no apparent lesions on CT scan. Malignancy of the disease is an important and feared complication, although tumors usually present with low grade, there are clinical signs of malignancy that should be monitored in these patients, such as the growth of exostosis after bone maturity, neuropraxia and symptoms associated with organ pressure close to injuries. 